\title{
THE INFLUENCE OF J. H. M. WEDDERBURN ON THE DEVELOPMENT OF MODERN ALGEBRA
}

It is obvious that the title of this paper is presumptuous. Nobody can give in a short article a really exhaustive account of the influence of Wedderburn on the development of modern algebra. It is too big an undertaking and would require years of preparation. In order to present at least a modest account of this influence it is necessary to restrict oneself rather severely. To this effect we shall discuss only the two most celebrated articles of Wedderburn and try to see them in the light of the subsequent development of algebra. But even this would be too great a task. If we would have to mention all the consequences and applications of his theorems we could easily fill a whole volume. Consequently we shall discuss only the attempts the mathematicians made to come to a gradual understanding of the meaning of his theorems and be satisfied just to mention a few applications.

For the understanding of the significance that Wedderburn's paper On hypercomplex numbers (Proc. London Math. Soc. (2) vol. 6, p. 77) had for the development of modern algebra, it is imperative to look at the ideas his predecessors had on the subject.

The most striking fact is the difference in attitude between American and European authors. From the very beginning the abstract point of view is dominant in American publications whereas for European mathematicians a system of hypercomplex numbers was by nature an extension of either the real or the complex field. While the Europeans obtained very advanced results in the classification of their special cases with methods that were not well adapted to generalization, the Americans achieved an abstract formulation of the problem, developed a very suitable terminology, and discovered the germs of the modern methods.

On the American side one has first of all to consider the very early paper by B. Peirce, Linear associative algebras (1870, published in Amer. J. Math. vol. 4 after his death). In it he states explicitly that mathematics should be an abstract logical scheme, the absence of a special interpretation of its symbols making it more useful in that the same logical scheme will in general reflect many diverse physical situations. Although it is true that he was actually able to introduce and treat only the general linear associative algebra over the complex field, yet he clearly had in mind much more, and it is his attitude which leads to the modern postulational method. In his treatment of algebras he gives a rational proof of the existence of an idempotent 
and employs the well known Peirce decomposition of an algebra relative to an idempotent. His results were put in a more readable form by H. E. Hawkes, On hypercomplex number systems (Trans. Amer. Math. Soc. vol. 3 (1902)). A correct definition of an associative algebra over an arbitrary field seems to be given for the first time by L. E. Dickson, Definitions of a linear associative algebra by independent postulates (Trans. Amer. Math. Soc. vol. 4 (1903)).

At that time several European mathematicians, Molien, Cartan, and Frobenius, had already arrived (always for the special cases of the real or complex field) at many of the results of the modern theory. The notions radical, semisimple, simple had been found and the decomposition of a semisimple algebra into simple components proved. Cartan derived the structure of the simple algebras but apparently without recognizing the possibility of stating the result in the very simple form Wedderburn discovered. It has to be borne in mind that all these authors had the complex field at their disposal and were therefore never hestitant to use roots of algebraic equations. This fact made a direct generalization of their results to arbitrary fields very difficult.

Wedderburn succeeded in a synthesis of these two lines of investigation. He extended the proof of all the structural theorems found by the European mathematicians for the special cases of the real and complex field to the case of an arbitrary field. By the effective use of a calculus of complexes (analogous to that which had been used in the treatment of finite groups) combined with the Peirce decomposition relative to an idempotent, he was able to prove his theorems within the given field and in a simpler way. He was the first to find the real significance and meaning of the structure of a simple algebra. We mean by this the gem of the whole paper, his celebrated:

"TheOREM 22-Any simple algebra can be expressed as the direct product of a primitive algebra and a simple matric algebra."

In his terminology primitive algebra means the same thing as what we now call division algebra.

This extraordinary result has excited the fantasy of every algebraist and still does so in our day. Very great efforts have been directed toward a deeper understanding of its meaning.

In the first period following his discovery the work consisted mainly in a polishing up of his proofs. But the fundamental ideas of all these later proofs are already contained in his memoir.

In the meantime a great change in the attitude of the algebraists had taken place. The European school had discovered the great ad- 
vantage of the abstract point of view which had been emphasized so early in the American school. The algebraists began to analyze Wedderburn's methods and tried to find an even more abstract background.

The essential point in the definition of an algebra is that it is a vector space of finite dimension over a field. This fact allows us to conclude that ascending and descending chains of subalgebras will terminate. After the great success that Emmy Noether had in her ideal theory in rings with ascending chain condition, it seemed reasonable to expect that in rings where the ascending and the descending chain condition holds for left ideals one should obtain results similar to those of Wedderburn. As one of the papers written from this point of view we mention E. Artin, Zur Theorie der hyperkomplexen Zahlen (Abh. Math. Sem. Hamburgischen Univ. vol. 5 (1926)). In 1939 C. Hopkins showed (Rings with minimal condition for left ideals, Ann. of Math. vol. 40) that the descending chain condition suffices.

Independently of Wedderburn's paper, the representation theory of groups had been developed under the leadership of Frobenius, Burnside, and I. Schur. These mathematicians had been very well aware of the connection with algebras, a connection given by the notion of a group ring. But little use was made of the theory of algebras.

It was Emmy Noether who made the decisive step. It consisted in replacing the notion of a matrix by the notion for which the matrix stood in the first place, namely, a linear transformation of a vector space.

Emmy Noether introduced the notion of a representation spacea vector space upon which the elements of the algebra operate as linear transformations, the composition of the linear transformations reflecting the multiplication in the algebra. By doing so she enables us to use our geometric intuition. Her point of view stresses the essential fact about a simple algebra, namely, that it has only one type of irreducible space and that it is faithfully represented by its operation on this space. Wedderburn's statement that the simple algebra is a total matrix algebra over a quasifield is now more understandable. It simply means that all transformations of this space which are linear with respect to a certain quasifield are produced by the algebra. This treatment of algebras may be found in van der Waerden's Moderne Algebra.

Recently it has been discovered that this last described treatment of simple algebras is capable of generalization to a far wider class of rings. 
One considers a ring $R$ and an additive group $V$ with $R$ as left operator domain $-V$ playing the role of the representation space and called $R$-space for short. Chevalley and Jacobson proved a direct generalization of Wedderburn's theorem if two simple axioms are satisfied: That the ring $R$ is faithfully represented by its action on $V$ and that $V$ is irreducible (this means that 0 and $V$ are the only $R$-subspaces of $V$ ). In these terms the proof is essentially simple and geometrical, no idempotents being required, and no finiteness assumption on $R$.

In homage to J. H. M. Wedderburn we present in fuller detail this modern proof of his theorem.

Let $R$ be a ring, $V$ an $R$-space satisfying the two axioms stated above. ${ }^{1}$ We shall show that $V$ is naturally a vector space over a certain quasifield $D$ and that practically all $D$-linear transformations of $V$ are produced by elements of $R$.

To construct the quasifield is easy. Let $D$ be the set of all homomorphisms of $V$ into itself. $D$ is a ring from first principles. Since the kernel of a nonzero element of $D$ is an $R$-subspace of $V$ which is different from $V$, this kernel is zero, and the element is an isomorphism. Since the image of $V$ under this isomorphism is an $R$-subspace of $V$ which is not zero, it is all of $V$, and we have an isomorphism of $V$ onto $V$. Such a map has an inverse and we see that $D$ is a quasifield. We have obtained in a natural, invariant manner the quasifield which Wedderburn obtained only in a noninvariant way as subring of $R$.

We denote the typical element of $D$ by $d$ and write these elements on the right of $V$ so that our space $V$ becomes now a right vector space over the quasifield $D$.

If $W$ is a $D$-subspace of $V$, then the set of all elements of $R$ which annihilate $W$ is a left ideal of $R$ which we shall call $W \#$. If $L$ is a left ideal of $R$, then the set of all elements of $V$ annihilated by $L$ is a $D$ subspace of $V$ which we shall denote by $L^{b}$.

We can now state and prove the fundamental lemma:

$$
\left(L \cap(\xi \cdot D)^{\#}\right)^{b}=L^{b}+\xi \cdot D
$$

for any left ideal $L$ of $R$ and any element $\xi$ of $V$.

Proof. The right-hand side is trivially contained in the left. If $L \xi=0$, the equation becomes $L^{b}=L^{b}$. It remains only to prove that the left-hand side is contained in the right under the assumption that $L \xi \neq 0$. Since $L \xi$ is a subspace of $V$, and $V$ is irreducible, $L \xi=V$ : every element of $V$ can be expressed in the form $l \xi$ where $l \in V$. Let $\eta$ be an

${ }^{1}$ I follow a presentation given by Mr. J. T. Tate. 
element of the left side of (1). It is annihilated by $L \cap(\xi D)^{\#}$, hence by every $l \in L$ which is in $(\xi \cdot D) \#$. $\eta$ is consequently annihilated by every $l \in L$ for which $l \xi=0$. Let now $\zeta$ be any element of $V$; if we write it in the form $\zeta=l \xi$ and map it onto the element $l \eta$ of $V$ we have before us a well defined map. If indeed $\xi=l \xi=l \xi_{1}$ then $\left(l-l_{1}\right) \xi=0$ hence $\left(l-l_{1}\right) \eta=0$ or $l \eta=l_{1} \eta$. That this map $l \xi \rightarrow l \eta$ is a homomorphism of $V$ into $V$ follows from the fact that $L$ is a left ideal; as such it is a certain element $d$ of $D$ and satisfies $(l \xi) d=l \eta$ for all $l \in L$. The element $\eta-\xi d$ is therefore annihilated by $L$ and is consequently an element of $L^{b}$. This shows

$$
\eta \in L^{b}+\xi \cdot D
$$

which is what we were trying to prove. It is of course the construction of the element $d$ which is the heart of this method.

Let $W$ be any $D$-subspace of $V$. If we substitute $L=W^{\#}$ in (1), the left side becomes

$$
\left(W^{b} \cap(\xi D)^{\#}\right)^{b} \text {. }
$$

Since obviously $(A+B)^{\#=A} \# \cap B^{\#}$ for any two $D$-subspaces $A$ and $B$ of $V$ we obtain from (1)

$$
(W+\xi D) \# b=W \# b+\xi D .
$$

This we can use to argue in the following manner.

$$
\text { If } W=W^{\# b} \text { then }(W+\xi D)=(W+\xi D) \text { \#b. }
$$

Combining a repeated application of (3) with

$$
0 \# b=0
$$

we obtain the

TheOREM.

$$
W_{0} \# b=W_{0}
$$

for any finite-dimensional $W_{0}=\xi_{1} D+\xi_{2} D+\cdots+\xi_{r} D$.

The only gap in the argument was the proof of (4): $0^{\# b}=$ (trivially) $R^{b}=$ an $R$-subspace of $V$ (which is not all of $V$ ) $=0$, again using the irreducibility of $V$.

Now let $\xi_{1}, \xi_{2}, \cdots, \xi_{r}$ be a finite number of elements of $V$, linearly independent over the quasifield $D$. Let $W=\xi_{1} D+\cdots+\xi_{r-1} D$. Since $\xi_{r} \notin W=W \#$ it follows that $W \# \cdot \xi_{r} \neq 0$. Therefore, as usual, by the irreducibility of $V$ we have $W^{\#} \cdot \xi_{r}=V$. Consequently there exists an element of $W \#$ which annihilates $\xi_{1}, \xi_{2}, \cdots, \xi_{r-1}$ and sends $\xi_{r}$ into 
any element of $V$. Combining such elements together we find an element of $R$ which sends the vectors $\xi_{i}$ independently into any set of $r$ elements of $V$. Viewing the $\xi_{i}$ as a basis for a finite-dimensional $D$ subspace $W_{0}$ of $V$ we see that any given $D$-linear map of $W_{0}$ into $V$ can be produced by an element of the ring $R$. This is what we meant by the statement that "practically all" $D$-linear maps of $V$ were produced by elements of $R$.

To specialize this result we must add the axiom that $R$ satisfies the descending chain condition on left ideals (this is obviously true if $R$ is Wedderburn's simple algebra). An ascending chain $W_{1} \subset W_{2} \subset \ldots$ of finite-dimensional $D$-subspaces of $V$ leads to a descending chain $W_{1}^{\#} \supset W_{2}^{\#} \ldots$ of left ideals of $R$ because of the statement $W \# b=W$. Therefore $V$ must satisfy the ascending chain condition on finitedimensional $D$-subspaces. This is possible only if $V$ itself is finitedimensional over $D$. In this case our previous result shows that every $D$-linear map of $V$ is produced by an element of $R$, and we have therefore obtained Wedderburn's theorem in geometric form.

As we have stated at the beginning it is not our intention to discuss the many applications Wedderburn's theorem has found, for instance, the investigations on division algebras by Wedderburn, Dickson, and others. They lead finally to a complete description of all simple algebras over an algebraic number-field by A. Albert, R. Brauer, H. Hasse, and Emmy Noether, or the theory of modular representations of algebras and groups by $\mathrm{R}$. Brauer.

Let us now consider the theorem of Wedderburn concerning finite fields ( $A$ theorem on finite algebras, Trans. Amer. Math. Soc. vol. 6 (1905)) and its influence on the development of modern algebra. One sees immediately that the characteristic of such a field $K$ is a prime $p>0$ and that the number of elements of $K$ is a power $p^{r}$ of $p$.

In $1903 \mathrm{E}$. H. Moore had determined all commutative fields of this type. The result was that to a given number $p^{r}$ of elements there exists (apart from isomorphisms) only one field, namely, the Galois field of degree $r$ and characteristic $p$. The proof for this fact was simplified considerably by Steinitz. It is his proof one finds in modern books on algebra.

In 1905 Wedderburn found the complete answer to our question in a paper entitled $A$ theorem on finite algebras, where he proves that every field with a finite number of elements is automatically commutative (under multiplication) and therefore a Galois field.

Wedderburn introduces the center $C$ of $K$ and also the normalizer $N_{\alpha}$ of any element $\alpha$ of $K$. It is obvious that $C$ and $N_{\alpha}$ are subfields and that $C \subset N_{\alpha}$ for each $\alpha$. Denoting by $q$ the number of elements of 
$C$ we find $q^{n_{\alpha}}$ resp. $q^{n}$ for the number of elements in $N_{\alpha}$ resp. $K$ where $n_{\alpha}$ and $n$ are the degrees of $N_{\alpha}$ resp. $K$ over $C$. Since $K$ is an extension of $N_{\alpha}$, the degree $n_{\alpha}$ divides $n$.

Wedderburn then considers the multiplicative group of $K$ of order $q^{n}-1$. He divides it into classes of conjugate elements and obtains an identity of the form:

$$
q^{n}-1=(q-1)+\sum_{n_{\alpha} \mid n_{n} n_{\alpha}<n} \frac{q^{n}-1}{q^{n_{\alpha}}-1}
$$

where he unites the classes with only one element in the term $q-1$ and where the sum runs over certain divisors $n_{\alpha}$ of $n$, the same divisors possibly several times.

In $\$ 4$ of his paper he shows the impossibility of (6) for $n>1$, making use of divisibility properties of numbers of the form $a^{n}-b^{n}$ which are hard to establish. In $\$ 5$ he gives another arrangement of this proof, again making use of these divisibility properties. A third proof by Dickson is based on similar ideas.

This result of Wedderburn has fascinated most algebraists to a very high degree and several attempts were made to simplify the proofs. Artin (Abh. Math. Sem. Hamburgischen Univ. vol. 5) gave a proof that did not make use of (6) and the divisibility properties but the proof is somewhat lengthy.

The first really simple proof of our theorem was given by E. Witt, Über die Kommutativität endlicher Schiefkörper in 1931 (Abh. Math. Sem. Hamburgischen Univ. vol. 8). Witt starts from (6) and makes the following simple remark:

If $\phi_{n}(x)$ is the $n$th cyclotomic polynomial, then each term in the sum on the right of (6) and also $q^{n}-1$ are obviously divisible by $\phi_{n}(q)$. Consequently $\phi_{n}(q) \mid q-1$. Since $\phi_{n}(q)=\mathrm{I}(q-\epsilon)$ where $\epsilon$ runs through the primitive $n$th roots of unity, we have $\phi_{n}(q)>q-1$ if $n>1$, and this shows the impossibility of $n>1$.

In 1933 a paper by C. C. Tsen, Divisionalgebren über Funktionenkörpern (Nachr. Ges. Wiss. Göttingen (1933)) shed a new light on the whole question. Tsen did not investigate finite fields, but he worked with algebraic fields $F$ of transcendency degree 1 with an algebraically closed field of constants. He proved that there does not exist any non-commutative extension field of finite degree. The method of his proof yielded really a much stronger theorem, namely:

If $N\left(x_{1}, x_{2}, \cdots, x_{n}\right)=0$ is an algebraic equation in $F$ without constant term and if the total degree $d$ is smaller than the number $n$ of unknowns $x_{i}$, there exists a nontrivial solution in $F$.

If one knows this theorem for a given field $F$ then $F$ cannot have 
any noncommutative extension field $E$ of finite degree. To see this let $\xi=x_{1} \omega_{1}+\cdots+x_{n} \omega_{n}$ be the generic element of $E\left(\omega_{1}, \cdots\right.$ a basis $)$ and let $N\left(x_{1}, x_{2}, \ldots, x_{n}\right)$ be the reduced norm in $E / F . N$ is a homogeneous form of $x_{1}, x_{2}, \cdots, x_{n}$ of a degree $d$ which is less than $n$ if $E$ is noncommutative. The theorem would give the existence of a $\xi \neq 0$ whose norm is 0 , which is a contradiction.

It occurred immediately to the mathematicians that possibly a Galois' field $F$ would have the same property, so that Wedderburn's theorem would appear as a consequence of a much more general theorem on Galois fields.

In 1935 C. Chevalley (Demonstration d'une hypothèse de M. Artin, Abh. Math. Sem. Hamburgischen Univ. vol. 11) proved this conjecture.

Wedderburn's theorem is therefore the special case of a more general Diophantine property of fields and thus has opened an entirely new line of research.

Emil Artin 